\title{
INFLUENCE DE L'ÉBULLITION DOMESTIQUE DU LAIT SUR SON EFFICACITÉ CALCIQUE
}

\author{
par \\ Denise HUGOT et JHAN CAUSERET
}

\section{I. - Introduction}

Le chauffage du lait entraîne l'insolubilisation partielle de certains sels de calcium solubles, en particulier du phosphate bicalcique : d'après Matrick et Halletw [5], une ébullition de une demi-heure abaisserait le taux du calcium diffusible à $3 \%$ du calcium total.

Bien que le suc gastrique puisse faire passer de nouveau sous forme soluble le calcium insolubilisé, on peut se demander si les traitements thermiques appliqués au lait pour le préserver ou le conserver ne risquent pas d'affecter sa valeur comme source de calcium.

Les résultats des principaux travaux consacrés au problème sont réunis dans le tableau I. Il existe entre ces résultats de nombreuses contradictions, qui peuvent d'ailleurs s'expliquer de bien des manières : origines différentes des laits comparés dans la même expérience, influence propre de la composition du régime alimentaire dans lequel est introduit le lait, variations des capacités d'absorption et de rétention du calcium avec l'espèce et en fonction de l'âge, grande diversité des techniques d'application d'un même traitement (1).

L'effet propre de l'ébullition domestique paraît n'avoir été étudié que par Daniels et Stearns [2]. Ces deux auteurs ont constaté, chez des enfants de 3 à 7 mois, que la rétention calcique et le coefficient de rétention du calcium étaient nettement plus élevés avec le lait bouilli rapidement (2), qu'avec le lait pasteurisé. Cependant, on peut faire à ces expériences, les critiques suivantes :

- les études métaboliques ont été de courte durée (3 jours pour chaque type de lait);

- elles ont porté sur des sujets dont le poids corporel était inférieur au poids moyen des enfants de même âge, et dont la rétention calcique était inférieure aux valeurs habituelles;

- l'efficacité calcique du lait bouilli a été comparée non à

(1) Les travaux cités dans le tableau I s'échelonnent sur une période de quarante ans (1916-1955), durant laquelle les techniques laitières ont considérablement évolué. Il parait done logique d'accorder plus de poids aux recherehes récentes qu'aux travaux anciens.

(2) Conditions et durée d'ébullition non précisées. 
TABLEAU I

INFLUENCE DES TRAITEMENTS THERMIQUES

SUR L'UTILISATION PHYSIOLOGIQUE DU GALGIUM DU LAIT

\begin{tabular}{|c|c|c|c|}
\hline Laits comparés & Sujets d'expérience & Résultats & Références \\
\hline Lait cru - lait pasteurisé ........ & Pores & Légère supériorité du lait cru & {$[4]$} \\
\hline Lait cru - lait pasteurisé (2) . . . . & Jeunes rats & Pas de différences & {$[1]$} \\
\hline Lait pasteurisé — lait bouilli ...... & Nourrissons & Nette supériorité du lait bouilli & {$[2]$} \\
\hline Lait cru - lait évaporé . . . . . . . . . & Jeunes porcs & Légère supériorité du lait eru & {$[10]$} \\
\hline Lait cru - lait évaporé . . . . . . . . . & Femmes adultes & Pas de différences & {$[3]$} \\
\hline Lait pasteurisé - lait évaporé ...... & Enfants de 3 à 12 ans & Nette supériorité du lait évaporé & [11] \\
\hline Lait pasteurisé $(1)$ - lait évaporé .... & Femmes adultes & Nette supériorité du lait évaporé & {$[3]$} \\
\hline Lait pasteurisé - lait évaporé $\ldots \ldots \ldots$ & Nourrissons & Pas de différences & [9] \\
\hline Lait cru - lait en poudre $\ldots \ldots \ldots \ldots$ & Enfants de 7 à 12 ans & Nette supériorité du lait cru & {$[3]$} \\
\hline Lait cru - lait en poudre ........ & Jeunes rats & Pas de différences & {$[8]$} \\
\hline Lait pasteurisé (1) - lait en poudre ... & Femmes adultes & Nette supériorité du lait pasteurisé & [3] \\
\hline Lait pasteurisé (3) - lait en poudre ... & Jeunes rats & Pas de différences & [6] \\
\hline
\end{tabular}

(1) Pasteurisation haute. - (2) Pasteurisation basse. - (3) Pasteurisation instantanée. 
celle du lait correspondant non bouilli, mais à celle d'un autre lait traité par la pasteurisation haute.

Ce dernier fait présente une grande importance, pour l'interprétation des résultats obtenus. Il s'y ajoute que, d'après DANIELS et STEARns, les enfants alimentés avec du lait traité par la pasteurisation haute se développent mal et ont un bilan calcique médiocre, parfois même négatif.

Les résultats obtenus suggèrent donc que la pasteurisation haute, telle qu'elle était appliquée il y a trente ans aux laits étudiés, altérait gravement leurs propriétés nutritionnelles, tandis qu'une ébullition "rapide" les respectait mieux. Depuis cette.date, les techniques de pasteurisation couramment appliquées ont beaucoup évolué, et il apparaît très vraisemblable que ni la pasteurisation basse, ni la pasteurisation instantanée, ne présentent d'inconvénients du même genre.

Les expériences de Daniels et Stearns ayant beaucoup perdu de leur intérêt, il nous a paru utile d'étudier comparativement l'efficacité calcique du lait, avant et après ébullition.

\section{II. - Techniques expérimentales}

L'expérience a porté sur 21 rats blancs, du sexe mâle, pesant initialement de 130 à 150 grammes. Durant 13 jours, ces animaux ont reçu un régime qui comprenait comme seule source de calcium, du lait pasteurisé non bouilli (lot $I$ ), le même lait bouilli 5 minutes dans un récipient en pyrex (lot II) ou le même lait bouilli dans les mêmes conditions et privé de sa "peau " (lot III). La composition des régimes était la suivante :

$$
\begin{aligned}
& \text { Lait . . . . . . . . . } 300 \mathrm{~cm}^{3} \\
& \text { Amidon .............. } 65 \mathrm{gr} \text {. } \\
& \text { Mélange salin spécial (1) ..... } 0 \text { gr. } 0075
\end{aligned}
$$

Les bilans calciques n'ont commencé que le quatrième jour et ont duré dix jours. Le calcium a été dosé par manganimétrie dans les régimes, dans les urines acidifiées par $\mathrm{ClH}$ et dans les fèces préalablement déshydratées et réduites en poudre.

Les sujets d'expérience ayant été utilisés également pour l'étude de l'influence de l'ébullition du lait sur son efficacité protéique, on trouvera dans le compte rendu de cette étude, les indications relatives à la présentation des régimes et au dispositif expérimental employé [7].

(1) Mélange de Hubbel et collaborateurs, réduit aux seules sources d'oligoéléments. 


\section{III. - Résultats}

Les résultats obtenus sont réunis dans le tableau $I I$.

L'utilisation du calcium a été évaluée par détermination du coefficient de rétention :

$$
\begin{gathered}
\text { C.R. }=\frac{\text { Ca ingéré }-(\text { Ca fécal }+ \text { Ca urinaire })}{\text { Ca ingéré }} \times 100 \\
\text { TABLEAU II }
\end{gathered}
$$

\begin{tabular}{|c|c|c|c|}
\hline Rats & $\frac{\text { Ca técal }}{\text { Ca excrété }} \times 100$ & $\frac{\text { Ca urinaire }}{\text { Ca excrété }} \times 100$ & $\begin{array}{l}\text { Coefficient de } \\
\text { rétention du } \\
\text { calcium }(\%)\end{array}$ \\
\hline \multicolumn{3}{|l|}{ Lait non bouilli : } & p. 100 \\
\hline $1 \ldots \ldots \ldots \ldots \ldots \ldots$ & 83 & 17 & 92 \\
\hline $2 \ldots \ldots \ldots \ldots \ldots \ldots \ldots$ & 69 & 31 & 92 \\
\hline $3 \ldots \ldots \ldots \ldots \ldots \ldots$ & 67 & 33 & 92 \\
\hline $4 \ldots \ldots \ldots \ldots \ldots \ldots$ & 55 & 45 & 90 \\
\hline $5 \ldots \ldots \ldots \ldots \ldots \ldots \ldots$ & 67 & 33 & 93 \\
\hline $6 \ldots \ldots \ldots \ldots \ldots \ldots$ & 63 & 37 & 91 \\
\hline $7 \ldots \ldots \ldots \ldots \ldots \ldots$ & 65 & 35 & 89 \\
\hline 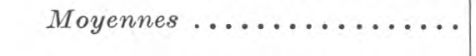 & 67 & 33 & $91 \cdot$ \\
\hline & $(\varepsilon= \pm 3,2)$ & $(\varepsilon= \pm 3,2)$ & $(\varepsilon= \pm 0,5)$ \\
\hline \multicolumn{4}{|l|}{ Lait bouilli, avec peau : } \\
\hline $8 \ldots \ldots \ldots \ldots \ldots \ldots$ & 53 & 47 & 90 \\
\hline $9 \ldots \ldots \ldots \ldots \ldots \ldots \ldots$ & 46 & 54 & 95 \\
\hline $10 \ldots \ldots \ldots \ldots \ldots \ldots \ldots$ & 48 & 52 & 92 \\
\hline $11 \ldots \ldots \ldots \ldots \ldots \ldots \ldots$ & 47 & 53 & 89 \\
\hline $12 \ldots \ldots \ldots \ldots \ldots \ldots \ldots$ & 65 & 35 & 93 \\
\hline $13 \ldots \ldots \ldots \ldots \ldots \ldots \ldots$ & 75 & 25 & 92 \\
\hline $14 \ldots \ldots \ldots \ldots \ldots \ldots \ldots$ & 68 & 32 & 91 \\
\hline $15 \ldots \ldots \ldots \ldots \ldots \ldots \ldots$ & 56 & 44 & 90 \\
\hline Moyennes .............. & $\begin{array}{c}57 \\
(\varepsilon= \pm 3,8)\end{array}$ & $\begin{array}{c}43 \\
(\varepsilon= \pm 3,8)\end{array}$ & $\begin{array}{c}91 \\
(\varepsilon= \pm 0,7)\end{array}$ \\
\hline \multicolumn{4}{|l|}{ Lait bouilli, peau enlevée : } \\
\hline $16 \ldots \ldots \ldots \ldots \ldots \ldots \ldots$ & 57 & 43 & 91 \\
\hline $17 \ldots \ldots \ldots \ldots \ldots \ldots \ldots$ & 54 & 46 & 93 \\
\hline $18 \ldots \ldots \ldots \ldots \ldots \ldots \ldots$ & 55 & 45 & 91 \\
\hline $19 \ldots \ldots \ldots \ldots \ldots \ldots \ldots$ & 73 & 27 & 91 \\
\hline $20 \ldots \ldots \ldots \ldots \ldots \ldots \ldots$ & 51 & 49 & 93 \\
\hline $21 \quad \ldots \ldots \ldots \ldots \ldots \ldots \ldots \ldots$ & 37 & 63 & 89 \\
\hline 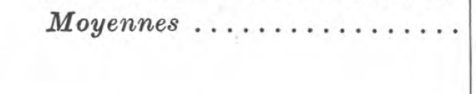 & $\begin{array}{c}55 \\
(\varepsilon= \pm 4,7)\end{array}$ & $\begin{array}{c}45 \\
(\varepsilon= \pm 4,7)\end{array}$ & $\begin{array}{c}91 \\
(\varepsilon= \pm 0,7)\end{array}$ \\
\hline
\end{tabular}

EFFIGAGITÉ GALGIQUE DU LAIT NON BOUILLI ET DU LAIT BOUILII 
Cette formule n'exprime que l'utilisation globale du calcium. Nous avons préféré ne pas calculer de coefficients d'utilisation digestive et de coefficients d'utilisation physiologique, la détermination de ces coefficients étant faussée par la présence possible, dans les fèces, d'une quantité de calcium endogène importante que la méthode ne permet pas d'évaluer. Par contre, nous avons indiqué les proportions du calcium excrété total qui sont éliminées par la voie intestinale et par la voie rénale.

On voit que la valeur moyenne du coefficient de rétention du calcium ainsi que la dispersion des valeurs individuelles autour de la moyenne, sont identiques pour les trois lots.

Les valeurs moyennes des rapports $C a$ fécal/Ca excrété total et Ca urinaire/Ca excrété total diffèrent pour ces mêmes lots. Mais, comme on peut le montrer par calcul, il est peu probable que les différences observées aient une signification : la dispension des valeurs individuelles suffit à les expliquer. Tout se passe comme si la capacité d'absorption du calcium variait beaucoup d'un sujet à un autre, l'élimination calcique rénale variant elle-même en sens inverse de la quantité de calcium absorbée et ramenant ainsi la rétention calcique à une valeur assez constante.

\section{Résumé}

L'ébullition du lait pasteurisé, réalisée pendant une durée de cinq minutes et suivie de l'enlèvement immédiat de la "peau" formée n'a entraîné, dans nos expériences, aucune modification sensible du coefficient de rétention du calcium de cet aliment.

(Laboratoire de Physiologie de la Nutrition, de l'Institut National de la Recherche Agronomique.)

\section{BIBLIOGRAPHIE}

[1] Commrtees on Milk and Nutrition. Milk and Nutrition, PartI, 1937, Reading.

[2] A. L. Dantels et G. Stmarns. Journal Biol. Chem, 1924, 61, 225.

[3] M. M. Kramer, E. Latzke ot M. M. SHAw. Journal Biol. Chem., 1928, 79, 283.

[4] H. E. MAGEE et D. HARvey. Biochem. Journal, 1926, 20, 885.

[5] E. C. V. Matitick et H. S. Hallemt. Journal Agric. Res., 1929, 19, 452.

[6] L. Randoin, D. Hugot et J. Causeret. Résultats non publiés.

[7] G. Reynaud et J. CaUseret. Le Lait, 1956, 36, 369.

[8] J. B. Shrelds, B. W. Fatrbanks, G. H. Berryman et H. H. MitOHELL. Journal Nutr, 1940, 20, 263.

[9] G. Stranns et P. C. Jeans. Proc. Soc, Exp, Biol. Med,, 1934-1935, $32,428$. 
[10] R. M. Washburn et C. H. Jones. Vermont Agric. Exp. Sta. Bull., $1916,195$.

[11] A. C. Willatd et K. Blunt. Journal Biol. Chem., 1927, 75, 251.

\title{
UNE MÉTHODE SINACIDE \\ DE DOSAGE DE LA MIATIÈRE GRASSE DU LAIT UTILISANT LE MATÉRIEL GERBER (1)
}

\author{
par \\ J. C. GODFRAIN \\ Professeur à l'Ecole Nationale Vétérinaire de Toulouse
}

Le dosage de la matière grasse du lait, demandé de plus en plus fréquemment aux laboratoires, depuis le paiement du lait aux producteurs en fonction du taux de cette matière grasse, entraîne, pour ces laboratoires, la nécessité d'un personnel ne réalisant que ce dosage. Ce personnel, pour des raisons financières très compréhensibles, ne peut pas être hautement qualifié, et il importe de lui confier une tâche simple et surtout dénuée de tout danger.

Cependant, de très nombreuses techniques de dosage proposées ne répondent pas à ces critères et la méthode Gerber, issue du procédé américain Babcok, utilisée dans l'ensemble des laboratoires français, si elle est rapide, n'est pas sans inconvénients. Le matériel nécessaire est relativement coûteux, mais surtout la manipulation d'acide sulfurique concentré a déjà donné lieu à des accidents dus, pour une bonne part, à la fragilité des butyromètres, au mode de bouchage de ceux-ci ainsi qu'au nombre d'opérations nécessaires. $\mathrm{Si}$ on ajoute que les appareils mesureurs automatiques de l'acide sulfurique sont loin d'être étanches (pertes d'acide sur les tables de travail; hydratation de l'acide dans le réservoir), on s'aperçoit que la mise au point d'une nouvelle méthode de dosage est souhaitable.

Le problème que nous avons cherché à résoudre était done le suivant : réaliser le dosage de la matière grasse à l'aide du matériel dont les laboratoires disposent couramment, sans utiliser les liqueurs caustiques.

Dans la résolution de ce problème, nous avons été guidé par les travaux de Schain et de Gershenfeld notamment. Les techniques suggérées par ces auteurs sont basées sur l'utilisation des détergents pour briser la couche protectrice des globules gras et permettre la formation d'une couche continue. Les détergents utilisés sont de deux types : l'un non ionisé, l'autre anionique.

(1) Revue de Médecine Vétérinaire, 1955, 106, 370. 